\title{
A MEDIÇÃO DO CWI E O PROCESSO DE DANIFICAÇÃO DE PARTÍCULAS DE ROCHAS*
}

Thaís Ferreira Marks Brasil Duque ${ }^{1}$ Claudio Luiz Schneider ${ }^{2}$

\section{Resumo}

O equipamento utilizado na medição do CWI de Bond também mede uma estimativa da energia de fratura das partículas de amostras de rochas e minérios. Esta medida só pode ser considerada uma estimativa da energia de fratura se, durante o ensaio, o material não é danificado. Em função do procedimento do ensaio de CWI, é possível que as partículas sejam danificadas por impactos sucessivos dos martelos em energias de impacto crescentes. Se houver danificação, a estimativa da energia de fratura será menor do que a energia de fratura real do material. Caso contrário a energia de fratura medida corresponde a energia de fratura mais a energia equivalente a um incremento de $5^{\circ}$ do ângulo de impacto. Neste trabalho uma metodologia foi desenvolvida especificamente para avaliar a danificação de partículas de rochas durante o ensaio de CWI. Quatro amostras distintas de rochas/minérios foram testadas. Os resultados mostram que há uma tendência consistente indicando que a danificação ocorre, porém, se de fato as partículas danificam, a magnitude da redução da energia de fratura é baixa ou incipiente.

Palavras-chave: Danificação; Britabilidade; Energia de fratura; Bond.

\section{THE MEASUREMENT OF BOND'S CWI AND THE PROCESS OF ROCK PARTICLE DAMAGING}

\begin{abstract}
The device that is used for the measurement of Bond's CWI also makes an estimate of the particle's fracture energy. This measurement can only be considered an estimate of the fracture energy if, during the test, the particles are not damaged. As the test is carried out by increasing impact energy up until the particle breaks, it is possible that damage is induced upon the the particles. In case damage is induced, the estimate of fracture energy is lower than the real fracture energy of the material. If damage is not induced than the measured fracture energy corresponds to the material fracture energy plus an energy increment equivalent of up to $5^{\circ}$ increase in the impact angle. In this work a procedure developed specifically to evaluate damage in rock particles during the Bond CWI test is presented. Four different rocks/ore types were tested. The results show that there is a consistent tendency towards damage, however, if in fact the particles are damaged, the magnitude of the reduction of fracture energy is low or insignificant.
\end{abstract}

Keywords: Damage; Crushability; Fracture energy; Bond.

1 Engenheira Química, M.Sc., Colaboradora, Coordenação de Processos Minerais, Centro de Tecnologia Mineral, Rio de Janeiro, Rio de Janeiro, Brasil.

2 Engenheiro de Minas, PhD., Tecnologista Sênior, Coordenação de Processos Minerais, Centro de Tecnologia Mineral, Rio de Janeiro, Rio de Janeiro, Brasil. 


\section{INTRODUÇÃO}

A medição do $C W I$ consiste em impactar partículas de rocha com energias crescentes até atingir uma energia de impacto maior que a energia de fratura da partícula em questão. Neste ponto a partícula é quebrada e o ângulo de inclinação dos martelos é registrado. Este ângulo é proporcional à energia de impacto. O valor do $C W I$ é medido desta forma para vinte partículas e a média é tomada como o valor do $C W I$ para a amostra.

Uma das questões que decorrem desta metodologia se refere à danificação das partículas durante o ensaio. Em geral, as partículas são impactadas mais de uma vez, sendo que todas as partículas sofrem pelo menos um impacto logo abaixo da sua energia de fratura (correspondente ao ângulo de inclinação em que a partícula efetivamente quebra menos $5^{\circ}$ ). Processos de danificação, na maioria das vezes, requerem esforços repetitivos e a danificação é cumulativa ao longo do tempo (número de esforços, impactos, eventos de danificação).

A medida que partículas de rochas sofrem cargas externas, as ligações químicas da microestrutura da rocha sofrem excitação em função de processos termodinâmicos. Esta excitação compõe a energia dada para a matriz da rocha ir além da energia de ativação das ligações e com isso as ligações começam a quebrar. Esta quebra de ligações é chamada de danificação (ENAYATPOUR; PATZEK, 2012).

No ensaio de Bond é comum impactar partículas entre 8 e 12 vezes em condições normais. Este número de impactos é baixo quando se considera processos de danificação, porém as energias específicas de impacto são relativamente altas durante o ensaio. Portanto, é possível que danificação ocorra durante o ensaio de CWI.

A ausência, ou presença, de danificação, pode ser medida, para o ensaio de CWI. Basta utilizar duas amostras de 20 partículas da rocha em questão. Um conjunto de partículas é utilizado para determinar o $C W I$ e a energia de fratura média da rocha. Essa energia média corresponde a um ângulo de impacto no equipamento. Se o segundo conjunto de partículas for testado com essa energia média espera-se que metade das partículas (10 partículas) quebre e a outra metade não quebre, já que $50 \%$ das partículas devem necessariamente ter energia de fratura menor do que a média e $50 \%$ maior do que a média. Porém, se um processo de danificação tiver ocorrido durante o ensaio, as partículas originais terão quebrado com uma energia menor do que a energia de fratura. Nesse caso em particular, o segundo conjunto de amostra apresentará menos do que $50 \%$ das partículas quebradas na energia média de impacto.

O objetivo primário deste trabalho é verificar, em diversas amostras de rochas, se o processo de danificação ocorre durante a medição do $C W I$ e se este processo pode ser detectado de alguma forma.

Em 1946, F. C. Bond detalhou o desenvolvimento de um dispositivo de impacto na forma de pêndulo que ainda é usado pelos principais fabricantes de britadores. $O$ dispositivo é constituído por duas rodas de bicicleta com martelos de aço tangencialmente ligados aos aros. Em cada extremidade dos cabos de elevação é ligado um martelo (Figura 1). $O$ peso dos martelos de aço pode ser diferente para cada tipo de amostra. A distância entre os martelos é de $50 \mathrm{~mm}$, quando estes estão em repouso. Uma rocha de tamanho entre $-76+50 \mathrm{~mm}$ é colocada sobre um suporte, de modo que os martelos atinjam a rocha simultaneamente, quando os cabos de elevação do martelo são liberados (MOORE, 1982). 


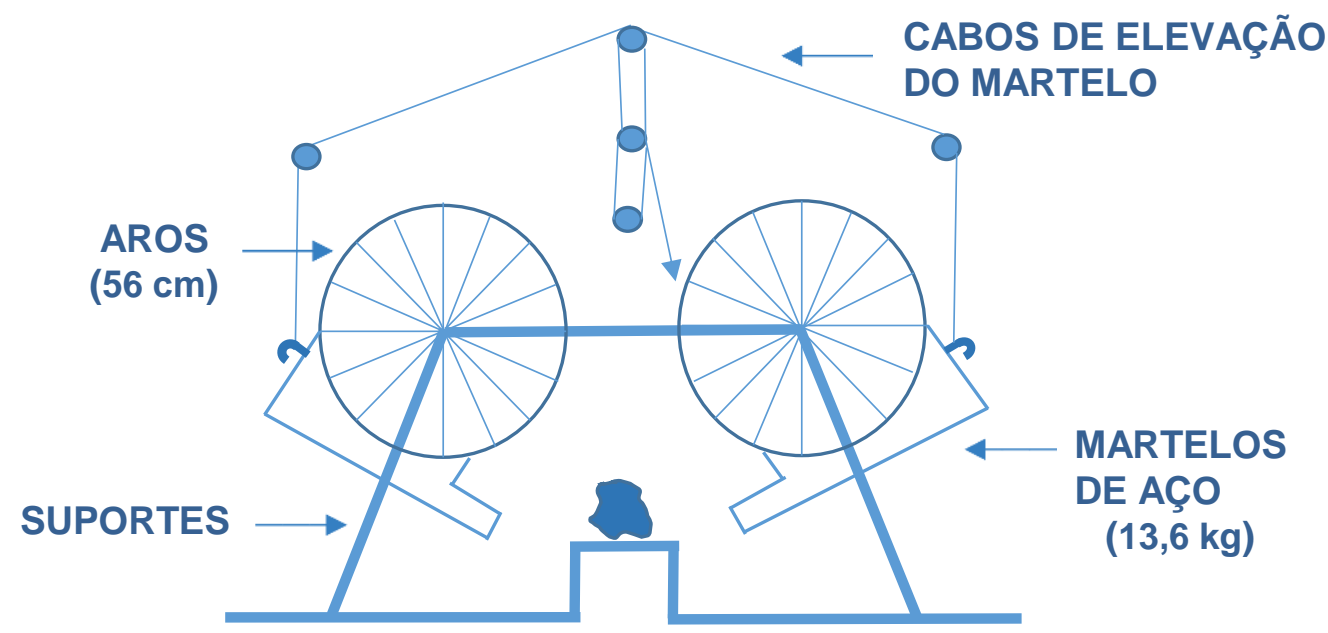

Figura 1. Equipamento de Bond - Pêndulo de Impacto (MOORE, 1982).

Uma vez que o procedimento de impacto de Bond foi desenvolvido não houve modificações no aparelho, procedimento ou análise de dados. Em geral, os resultados de britabilidade do ensaio de impacto de Bond estimam corretamente os parâmetros operacionais de britagem (MOORE, 1982).

As vantagens do ensaio de impacto de Bond são: não é necessária nenhuma preparação da amostra, a não ser o peneiramento de partículas de $-76+50 \mathrm{~mm}$; e, a análise dos resultados é simples. As desvantagens desse ensaio são: os resultados são imprecisos para britadores intermediários e finos; a variação na quebra da rocha individual é grande; não é possível fazer uma estimativa do tamanho do produto que será gerado pelo britador; e, o operador deve fazer julgamentos subjetivos referentes a quando uma rocha quebra, isto é, julgar se um pequeno fragmento representa ou não a quebra da partícula.

\section{MATERIAIS E MÉTODOS}

Nesse trabalho foram medidos os valores de CWI das seguintes rochas: Minério de Cobre, Minério de Manganês, Quartzo e Nefelina Sienito.

Dois conjuntos de vinte partículas de $-76 \mathrm{~mm}+50 \mathrm{~mm}$ de cada rocha foram escolhidos para os ensaios de impacto. A espessura de cada uma das partículas foi medida antes do ensaio. A densidade real das amostras também foi medida antes do ensaio.

Após o preparo das amostras, cada partícula foi colocada sobre um suporte e, então, os martelos foram elevados até $5^{\circ}$ e liberados, de modo que eles atingissem a partícula simultaneamente. Se a partícula não quebrasse, os martelos eram elevados novamente, com um incremento adicional de $5^{\circ}$, e liberados novamente. Esta operação foi realizada até que a partícula quebrasse. (Sempre que houvesse dúvida sobre a quebra ou não da partícula, o ângulo era incrementado em 5 ํa até que a quebra fosse identificada de forma inequívoca).

Foram realizados ensaios com martelos de aço de $13,62 \mathrm{~kg}$ com as amostras de: Quartzo e Nefelina Sienito e com martelos de $15,65 \mathrm{~kg}$ com as amostras de Minério de Cobre e de Minério de Manganês.

O CWI do CETEM foi desenvolvido pela empresa Top Size Soluções. Na Figura 2 é apresentado este equipamento. 


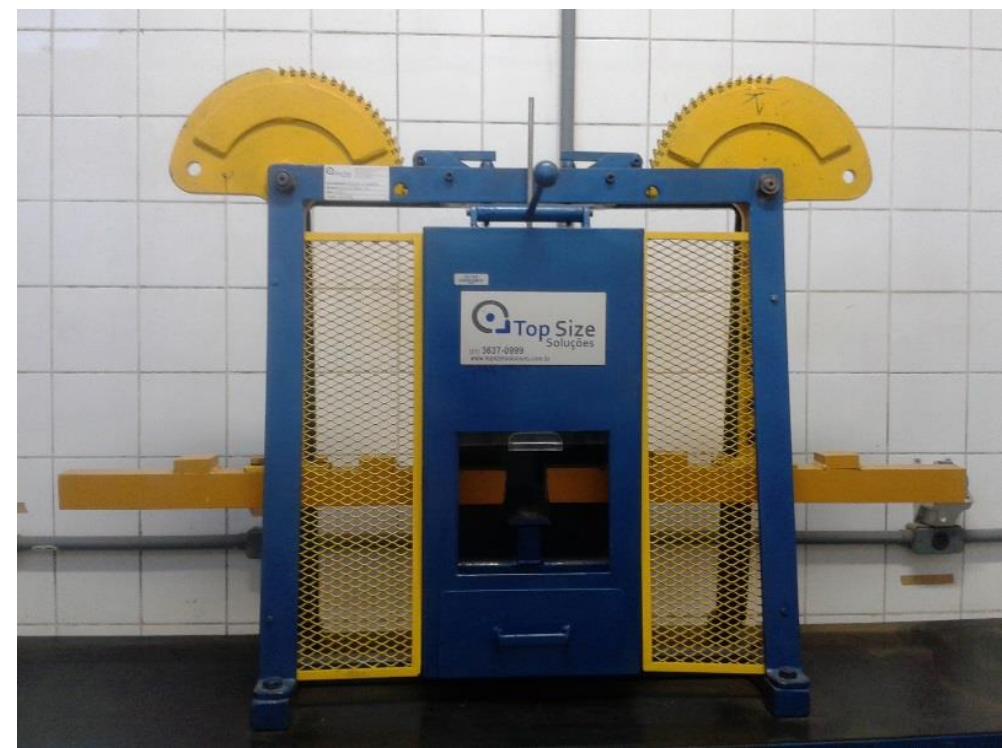

Figura 2. Equipamento de $C W I$ instalado no CETEM.

Os ensaios de impacto foram realizados de duas formas distintas para cada amostra: na primeira o ensaio foi realizado até que cada partícula fosse fragmentada, utilizandose o procedimento padrão do ensaio de CWI. Na segunda foi utilizado o valor de energia de fratura média necessário para que as 20 partículas quebrassem na primeira etapa.

A energia de fratura é uma função da altura de queda dos martelos e pode ser calculada para cada partícula utilizando a equação (1):

onde:

$$
E=2 \times H \times g \times P
$$

E é a energia de fratura, (Joule);

$H$ é a altura de queda do martelo, $(\mathrm{m})$;

$g$ é a aceleração da gravidade, igual a $9,81 \mathrm{~m} / \mathrm{s}^{2}$;

$P$ é o peso de cada martelo $(\mathrm{kg})$.

Por sua vez, a altura de queda do martelo é uma função do ângulo de giro do braço. $H$ pode ser calculada pela equação (2):

$$
H=(1-\cos \alpha) \times L
$$

onde:

$\alpha$ é o ângulo de giro do braço (rad);

$L$ é o comprimento do braço do martelo $(\mathrm{m})$.

Aqui cabe observar que, o comprimento $L$ é definido como a distância do centro de giro até o centro de massa do martelo. No equipamento do CETEM, $L=41,50 \mathrm{~cm}=$ $0,415 \mathrm{~m}$. Para que o equipamento funcione bem e para que os cálculos sejam acurados, a linha do raio de giro deve, necessariamente, passar pelo centro de massa do martelo. É, portanto mais recomendável fabricar martelos simétricos, pois desta forma a determinação do centro de massa é fácil por coincidir com o centro geométrico. É possível construir martelos de pesos diferentes, mais leves ou mais pesados, e, dependendo do material a ser testado, pode-se utilizar um peso mais apropriado. Materiais mais friáveis, com CWI baixo podem ser testados com martelos 
mais leves enquanto que rochas mais tenazes com $\mathrm{CWI}$ alto podem ser testados com martelos mais pesados.

O índice de britabilidade de Bond, CWI (kWh/t), de cada partícula é calculado em função da sua espessura e densidade, e da energia de fratura, como apresentado na equação (3):

onde:

$$
C W I=53,49 \times \frac{E}{\rho \times t}
$$

$t$ é a espessura de cada partícula $(\mathrm{mm})$

$\rho$ é a densidade real da rocha $\left(\mathrm{g} / \mathrm{cm}^{3}\right)$;

O CWI da amostra é a média dos vinte índices determinados para cada uma das vinte partículas da amostra.

As densidades medidas e o peso do martelo utilizado nos ensaios são mostrados na Tabela 1.

Tabela 1. Densidade dos minérios e rochas utilizados nos ensaios de Impacto e peso dos martelos

\begin{tabular}{ccc}
\hline Amostra & Densidade $\left(\mathbf{g} / \mathbf{c m}^{\mathbf{3}}\right)$ & Peso do martelo $\mathbf{( k g )}$ \\
\hline Quartzo & 2,561 & 13,62 \\
\hline Nefelina Sienito & 2,544 & 13,62 \\
\hline Minério de Cobre & 3,392 & 15,65 \\
\hline Minério de Manganês & 3,536 & 15,65 \\
\hline
\end{tabular}

\section{RESULTADOS E DISCUSSÃO}

Os resultados dos ensaios, segundo a metodologia de determinação do $C W I$ de Bond, são apresentados na Tabela 2. 
Tabela 2. Resultados do ensaio de determinação do índice de britabilidade de Bond

\begin{tabular}{|c|c|c|c|c|c|c|c|c|}
\hline \multirow{2}{*}{$\begin{array}{l}\text { Amostra } \\
\text { Partícula }\end{array}$} & \multicolumn{2}{|c|}{ Quartzo } & \multicolumn{2}{|c|}{ Nefelina Sienito } & \multicolumn{2}{|c|}{ Minério de Cobre } & \multicolumn{2}{|c|}{$\begin{array}{l}\text { Minério de } \\
\text { Manganês }\end{array}$} \\
\hline & $\begin{array}{c}t, \\
(\mathbf{m m})\end{array}$ & $\begin{array}{l}\text { Ângulo de } \\
\left.\text { Fratura, ( }{ }^{\circ}\right)\end{array}$ & $\underset{(\mathrm{mm})}{\boldsymbol{t}}$ & $\begin{array}{l}\text { Ângulo de } \\
\left.\text { Fratura, ( }{ }^{\circ}\right)\end{array}$ & $\underset{(\mathrm{mm})}{\boldsymbol{t}}$ & $\begin{array}{l}\text { Ângulo de } \\
\left.\text { Fratura, ( }{ }^{\circ}\right)\end{array}$ & $\underset{(\mathrm{mm})}{\boldsymbol{t}}$ & $\begin{array}{l}\text { Ângulo de } \\
\text { Fratura, ( }()\end{array}$ \\
\hline 1 & 65,0 & 55 & 45,0 & 50 & 41,0 & 25 & 62,0 & 50 \\
\hline 2 & 57,0 & 55 & 56,0 & 40 & 24,0 & 15 & 50,0 & 50 \\
\hline 3 & 58,0 & 55 & 40,0 & 35 & 53,0 & 70 & 63,0 & 25 \\
\hline 4 & 43,0 & 40 & 60,0 & 75 & 63,0 & 65 & 45,0 & 35 \\
\hline 5 & 52,0 & 50 & 62,0 & 70 & 54,0 & 90 & 49,0 & 30 \\
\hline 6 & 58,0 & 30 & 42,0 & 25 & 62,0 & 90 & 42,0 & 35 \\
\hline 7 & 48,0 & 35 & 45,0 & 35 & 41,0 & 75 & 31,0 & 15 \\
\hline 8 & 69,0 & 35 & 38,0 & 25 & 41,0 & 60 & 50,0 & 25 \\
\hline 9 & 62,0 & 55 & 37,0 & 45 & 45,0 & 55 & 53,0 & 45 \\
\hline 10 & 69,0 & 35 & 53,0 & 50 & 31,0 & 50 & 54,0 & 40 \\
\hline 11 & 45,0 & 50 & 43,0 & 30 & 44,0 & 35 & 52,0 & 25 \\
\hline 12 & 55,0 & 50 & 30,0 & 25 & 38,0 & 35 & 48,0 & 25 \\
\hline 13 & 59,0 & 25 & 19,0 & 20 & 54,0 & 75 & 44,0 & 40 \\
\hline 14 & 60,0 & 50 & 33,0 & 30 & 52,0 & 70 & 56,0 & 35 \\
\hline 15 & 67,0 & 70 & 35,0 & 30 & 44,0 & 65 & 56,0 & 45 \\
\hline 16 & 51,0 & 55 & 39,0 & 35 & 48,0 & 60 & 67,0 & 70 \\
\hline 17 & 52,0 & 35 & 51,0 & 40 & 55,0 & 35 & 70,0 & 55 \\
\hline 18 & 63,0 & 55 & 41,0 & 65 & 48,0 & 65 & 46,0 & 35 \\
\hline 19 & 66,0 & 40 & 32,0 & 25 & 71,0 & 50 & 50,0 & 30 \\
\hline 20 & 40,0 & 55 & 40,0 & 45 & 65,0 & 75 & 40,0 & 45 \\
\hline Média & & 46,5 & & 40 & & 58 & & 38 \\
\hline $\begin{array}{l}\text { Desvio } \\
\text { Padrão }\end{array}$ & & 11,37 & & 15,68 & & 20,55 & & 12,82 \\
\hline Mediana & & 50 & & 40 & & 62,5 & & 35 \\
\hline
\end{tabular}

Os valores de $C W I$ obtidos para essas amostras de rochas e minérios no ensaio de britabilidade de Bond estão apresentados na Tabela 3.

Tabela 3. CWI das rochas - Ensaio de Impacto de Bond padrão

\begin{tabular}{cc}
\hline Amostra & $\boldsymbol{C W I} \mathbf{( k W h / t )}$ \\
\hline Quartzo & 15,90 \\
\hline Nefelina Sienito & 12,77 \\
\hline Minério de Cobre & 19,89 \\
\hline Minério de Manganês & 8,10 \\
\hline
\end{tabular}

Os resultados da Tabela 2 podem ser utilizados para prever o comportamento das amostras na hipótese de não existir qualquer processo de danificação. Se esta hipótese estiver correta, espera-se que em torno de $50 \%$ das partículas quebrariam se tivessem sido testadas com o ângulo de impacto mediano resultante. A mediana é definida como uma estatística ou um número que separa a metade mais alta de uma população da metade mais baixa. Assim, espera-se que, por exemplo, $50 \%$ das 
partículas de Quartzo, ou seja, 10 partículas, quebrem com um ângulo de impacto igual a 50․ Na verdade esta divisão perfeita só pode ocorrer para incrementos de ângulo de impacto muito pequenos e um número de partículas testadas muito grande. Na prática, o ensaio de Bond prevê um número de partículas relativamente pequeno e um incremento de ângulo de impacto muito grande, igual a 5‥ Isso faz com que o número de partículas quebradas com o ângulo de impacto mediano não seja necessariamente igual a 50\%. Por exemplo, analisando os dados para as partículas de quartzo, se todas as partículas tivessem sido testadas com o ângulo igual a $50^{\circ}$, que é o valor da mediana, chegaríamos à conclusão de que 12 quebrariam e 8 permaneceriam intactas. Isso ocorre porque quatro partículas têm ângulo de fratura exatamente igual a $50^{\circ}$, e a metade superior da população inclui duas destas partículas. O caso do minério de cobre é ainda mais complexo porque o valor da mediana é um valor entre os ângulos de fratura de $60^{\circ}$ e $65^{\circ}$. Simbolicamente o valor da mediana é $62,5^{\circ}$, pois todas as partículas da metade superior têm ângulo de fratura maior ou igual a $65^{\circ}$ e todas as partículas da metade inferior tem ângulo de fratura menor ou igual a $60^{\circ}$. O problema aqui é que $62,5^{\circ}$ não é um ângulo de impacto possível no equipamento. $O$ critério utilizado nestes casos é considerar o ângulo múltiplo de $5^{\circ}$ imediatamente abaixo da mediana, ou seja, $60^{\circ}$ para o caso de minério de cobre. Se o critério utilizado é que a partícula quebra se for impactada com um ângulo maior ou igual a 60, chegaríamos a 10 partículas quebradas.

Adotando estes critérios, na hipótese de ausência de processos de danificação, o número de partículas quebradas previsto para as amostras de quartzo, nefelina sienito, minério de cobre e manganês, considerando-se os resultados da Tabela 2 , seriam 12, 11, 10 e 11, respectivamente. Embora os resultados estejam perto de 50\% da população (10 partículas) em todos os casos, nenhum caso prevê um número menor do que $50 \%$ das partículas quebradas. Isto ocorre porque o número de partículas testadas é finito e relativamente baixo, podendo ocorrer que mais de uma partícula apresente ângulo de fratura igual à mediana.

Após os ensaios de britabilidade de Bond, foram testadas mais vinte partículas de cada rocha, utilizando o valor do ângulo de impacto de fratura mediano do ensaio original, arredondado para o próximo ângulo, múltiplo de $5^{\circ}$, imediatamente abaixo, disponível para teste. Ou seja, o mesmo critério foi utilizado para a execução da parte experimental. Para estas amostras os seguintes valores de ângulo de impacto foram utilizados: $50^{\circ}$ para a amostra de quartzo, $40^{\circ}$ para a amostra de Nefelina Sienito, $60^{\circ}$ para a amostra de minério de cobre e $35^{\circ}$ para a amostra de minério de manganês. Os resultados desses ensaios são apresentados na Tabela 4. 
Tabela 4. Partículas ensaiadas com o ângulo de impacto médio de fratura

\begin{tabular}{|c|c|c|c|c|}
\hline Amostra & Quartzo & $\begin{array}{l}\text { Nefelina } \\
\text { Sienito }\end{array}$ & $\begin{array}{l}\text { Minério de } \\
\text { cobre }\end{array}$ & $\begin{array}{l}\text { Minério de } \\
\text { manganês }\end{array}$ \\
\hline 1 & Não & Sim & Sim & Não \\
\hline 2 & Sim & Sim & Não & Não \\
\hline 3 & Não & Sim & Sim & Não \\
\hline 4 & Sim & Sim & Não & Sim \\
\hline 5 & Sim & Não & Não & Não \\
\hline 6 & Sim & Não & Sim & Sim \\
\hline 7 & Não & Não & Não & Sim \\
\hline 8 & Não & Sim & Sim & Não \\
\hline 9 & Não & Não & Sim & Não \\
\hline 10 & Não & Sim & Sim & Sim \\
\hline 11 & Sim & Não & Sim & Sim \\
\hline 12 & Não & Sim & Sim & Não \\
\hline 13 & Sim & Sim & Não & Sim \\
\hline 14 & Sim & Não & Não & Não \\
\hline 15 & Sim & Não & Não & Não \\
\hline 16 & Sim & Não & Não & Não \\
\hline 17 & Não & Sim & Não & Sim \\
\hline 18 & Não & Sim & Não & Sim \\
\hline 19 & Não & Não & Não & Sim \\
\hline 20 & Sim & Não & Não & Não \\
\hline $\begin{array}{c}\text { Total } \\
\text { quebradas }\end{array}$ & 10 & 10 & 8 & 9 \\
\hline
\end{tabular}

Devemos salientar que, os resultados mostrados na Tabela 4 indicam que, no laboratório, um menor número de partículas quebrou quando comparado ao valor esperado na hipótese de não danificação. Onde tínhamos previsto 12, 11, 10 e 11 partículas quebradas foi obtido 10, 10, 8 e 9 partículas quebradas no ensaio de laboratório com as amostras de quartzo, nefelina sienito, minérios de cobre e de manganês.

\section{CONCLUSÃO}

Os resultados são mostrados de forma resumida na Figura 3. A previsão do número de partículas quebradas com o ângulo de fratura mediano na hipótese de ausência de processos de danificação é comparada com os resultados de laboratório para um único impacto com o ângulo mediano de fratura para as quatro amostras testadas. Em todos os casos o número de partículas quebradas no laboratório foi menor do que o número previsto. Estes resultados levam à conclusão que as partículas de minério são danificadas durante o ensaio de britabilidade de Bond.

É claro que a diferença não é dramática, como é apresentado na Figura 3. Também se deve levar em consideração que os dados aqui apresentados são ainda incipientes e que provavelmente um maior número de partículas e de minérios, assim como ensaios mais detalhados com intervalos de ângulos de impacto menores poderão levar à outras conclusões. No entanto, os resultados aqui apresentados servem como 
base para pelo menos promover a discussão e, em outro momento, a investigação dos processos de danificação em partículas de minério e rochas em geral.

Se a danificação realmente ocorreu nas quatro amostras testadas, isso implica que estes processos podem decorrer depois de um número limitado de esforços e não apenas em situações de esforços continuados.

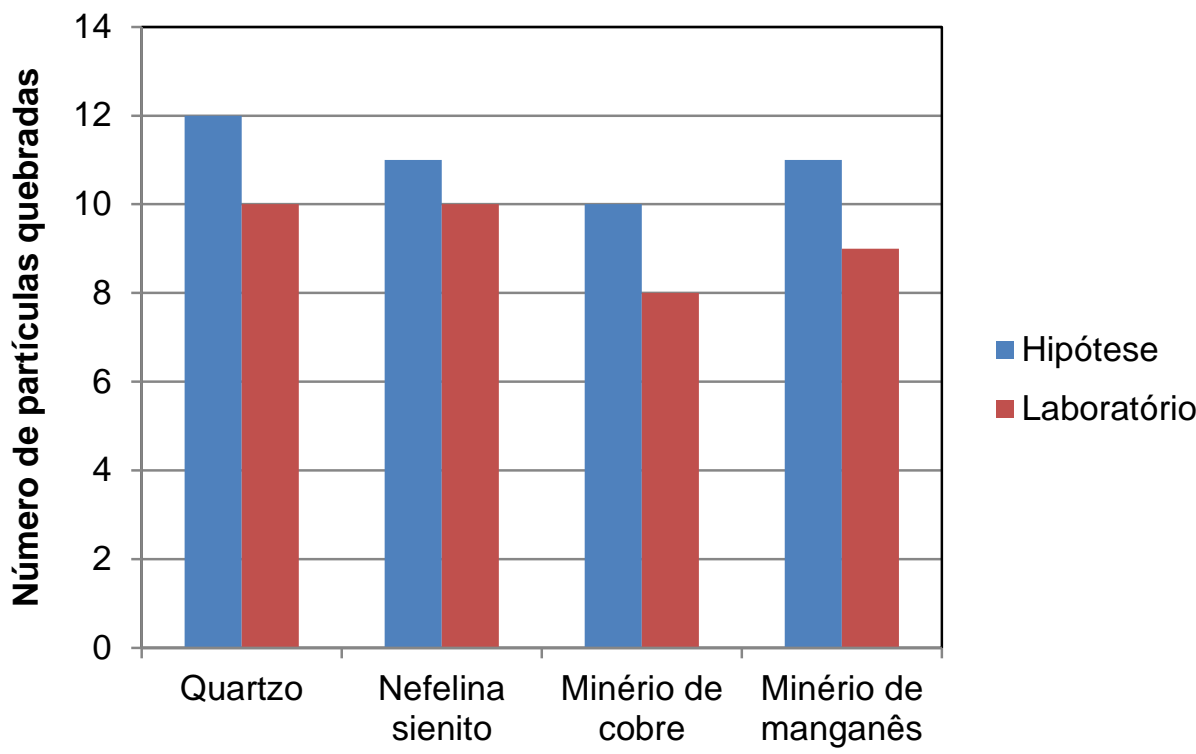

Figura 3. Número de partículas quebradas no ângulo de quebra previsto na hipótese de ausência de danificação e número de partículas efetivamente quebradas experimentalmente.

\section{Agradecimentos}

Os autores agradecem o financiamento continuado da empresa Norsk-Hydro para desenvolvimento de modelos e ensaios em escala piloto de plantas de moagem SAG. Também agradecemos por recursos do CNPq, Fundo CT-Mineral, Projeto 550270/2011-6.

\section{REFERÊNCIAS}

1 ENAYATPOUR, S.; PATZEK, T. "Finite Element Solution of Nonlinear Transient Rock Damage with Applications in Geomechanics of Oil and Gas Reservoirs", Proceedings of the 2012 COMSOL Conference, Boston, 2012.

Disponível em: www.comsol.com/paper/13893

$2 \quad$ BOND, F.C., "Crushing Tests by Pressure and Impact", Trans. AIME, Vol. 169, pp. 5865, 1947.

3 MOORE, D.C., "Prediction of Crusher Power Requirements and Product Size Analysis", Chapter 14, pp. 218-227. In: Design and Installation of Comminution Circuits, Mular A.L. \& Jergensen, G.V. (Editors), SME-AIME, New York, 1982. 